\title{
Photoconversion of Lysotracker Red to a green fluorescent molecule
}

\author{
Eric C Freundt ${ }^{1,3}$, Meggan Czapiga ${ }^{2}$, Michael J Lenardo ${ }^{1}$ \\ ${ }^{1}$ Laboratory of Immunology, ${ }^{2}$ Biological Imaging Section, Research Technologies Branch, National Institute of Allergy and Infectious \\ Diseases, National Institutes of Health, Bethesda, MD 20892, USA ${ }^{3}$ Medical Research Council Human Immunology Unit, Weatherall \\ Institute of Molecular Medicine, John Radcliffe Hospital, University of Oxford, Oxford OX3 9DS, UK
}

Cell Research (2007) 17:956-958. doi: 10.1038/cr.2007.80; published online 25 September 2007

\section{Dear Editor:}

Lysotracker Red DND-99 (Invitrogen-Molecular Probes) is a fluorophore in the form of a conjugated multi-pyrrole ring structure containing a weakly basic amine that selectively accumulates in acidic compartments and exhibits red fluorescence (excitation: $577 \mathrm{~nm}$, emission: $590 \mathrm{~nm}$ ) (Figure 1A). It is structurally related to Lysotracker Green (Figure 1B) but has an additional pyrrole ring in conjugation with the primary structure, which produces a longer wavelength emission. Lysotracker Red is commonly used in multicolor imaging studies as a lysosomal marker to determine intracellular localization of a protein of interest by fluorescence and confocal microscopy [1-5] and is recommended by the manufacturer for this application. While using Lysotracker Red to study the localization of a protein fused to green fluorescent protein (GFP), we observed an additional strong green fluorescent signal that colocalized with Lysotracker Red. After careful examination, however, we noted that the added green signal appeared only after illumination of the cells by a standard $100 \mathrm{~W}$ mercury epifluorescence light source equipped with a 560/40 excitation filter (Leica TX2). Prior to exposing the field to broadband excitation light, it was possible to visualize by confocal scanning (488nm excitation line) cells that exclusively expressed GFP. Remarkably, after exposure to broadband excitation light, green fluorescence appeared in all cells irrespective of GFP expression, displayed signal intensity similar to that of GFP, and colocalized with Lysotracker Red (Figure S1).

Correspondence: Michael J Lenardo

Tel: +1-301-496-6754; FAX: +1-301-402-8530

E-mail:mlenardo@niaid.nih.gov
This phenomenon was then reproduced in non-transfected cells that did not express GFP. Cells stained with Lysotracker Red according to manufacturer's recommendations did not initially display green fluorescence (Figure 1D, I) and showed appropriate Lysotracker Red staining and fluorescence (Figure 1E, J). After epifluorescent excitation, however, cells exhibited an intense green fluorescence that colocalized with the red signal (Fig 1F, K). In order to investigate the relationship of Lysotracker Red to the emergent green fluorescence, we compared the mean fluorescent signal obtained by excitation with a $488 \mathrm{~nm}$ laser or a $594 \mathrm{~nm}$ laser, with and without pre-exposure to broadband excitation, in cells stained with Lysotracker Red. By exciting Lysotracker Red with epifluorescence for one-second intervals, we observed a subsequent decay in mean red fluorescence intensity, which coincided with an increase in detectable green fluorescence (Figure 1M).

We sought to further characterize this phenomenon by obtaining the emission spectra of Lysotracker Red both before and after inducing conversion. The spectral properties of the dye were dramatically altered after induction. Preexposed dye was not excited by a $488 \mathrm{~nm}$ laser and showed no emission in the 500-575 $\mathrm{nm}$ spectrum. After conversion, however, Lysotracker Red exhibited an emission spectrum that was remarkably similar to that of Lysotracker Green (Figure 1N). Since the molecular structures of Lysotracker Red and Lysotracker Green are related (Figure 1A, B), it is plausible that excitation of the red-emitting molecule with broadband illumination induces molecular changes rendering its photochemical properties similar to those of the green-emitting molecule. The similarities between the spectra of Lysotracker Green and converted Lysotracker Red suggest that the third pyrrole ring has been taken out 
of conjugation leading to a shorter wavelength emission (Figure 1A, asterisk). We speculate that this occurs as a result of a double bond shift or a reduction. Additionally, we found that the spectral shift of Lysotracker Red is transient, reverting to native form within 30 minutes after conversion. This phenomenon was not cell-type specific and occurred in both live cells and cells fixed with 3.7\% paraformaldehyde, eliminating the possibility that cellular enzymes contributed to spectral shift of the molecule (data not shown).

It is surprising that this phenomenon has, to our knowledge, gone unreported in the literature given the frequency
A

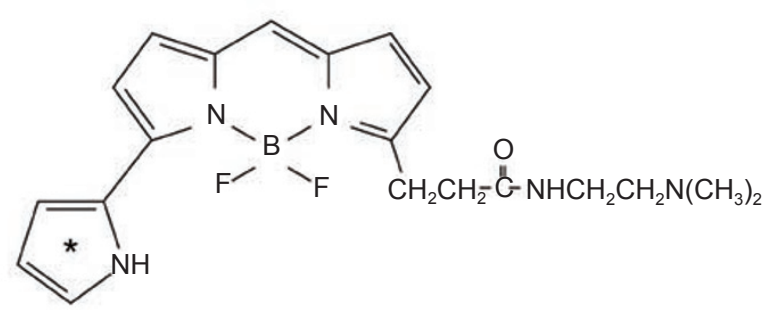

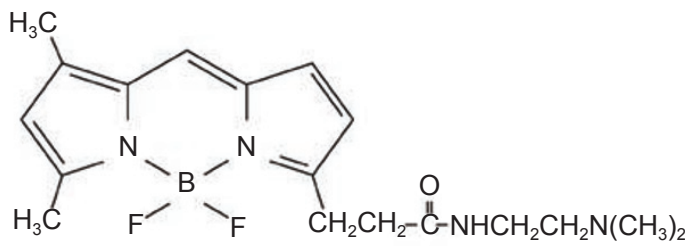
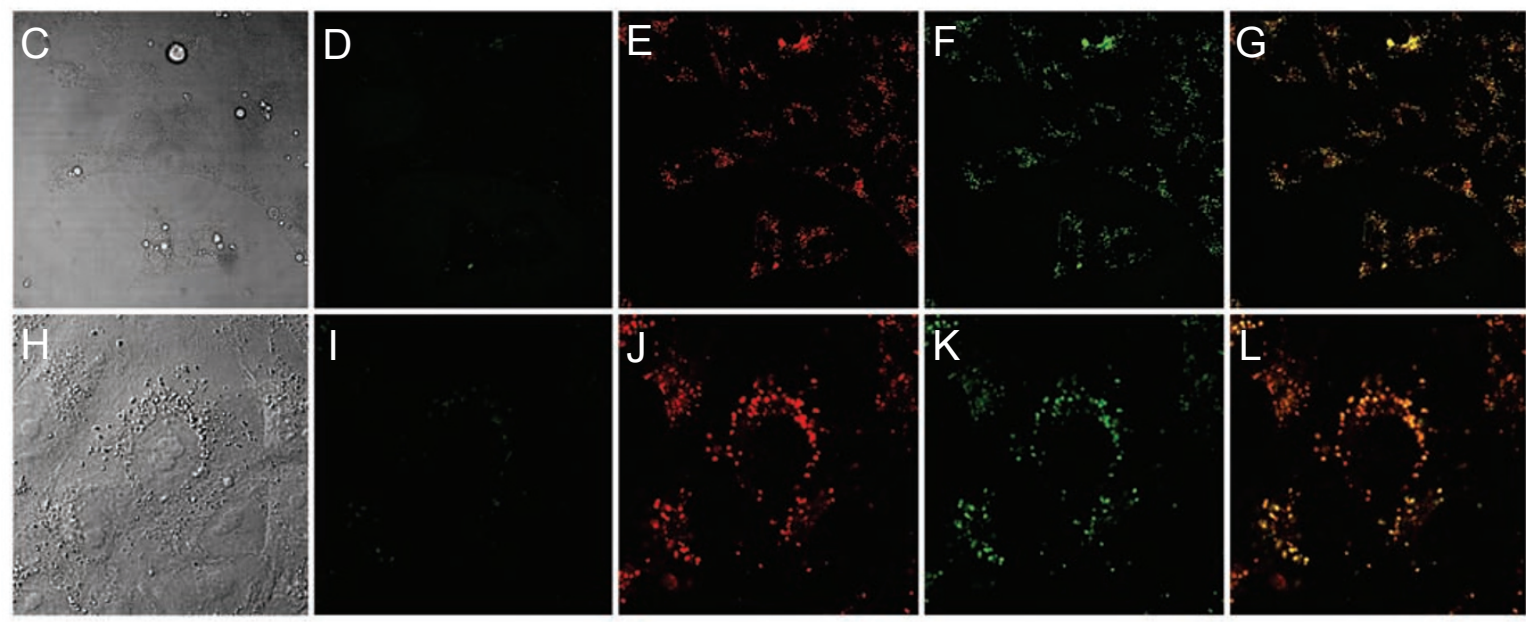

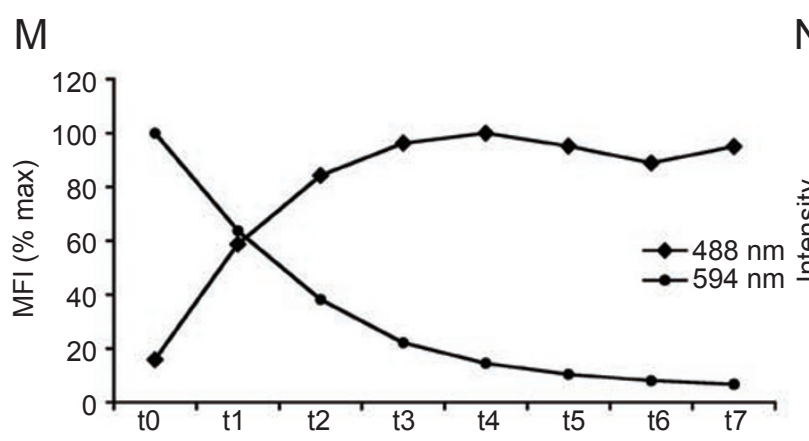

$\mathrm{N}$

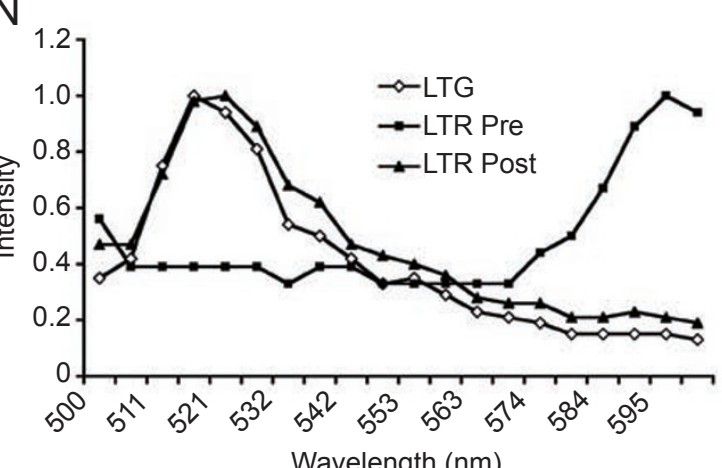

Figure 1 Lysotracker Red exhibits green fluorescence after excitation. The molecular structures of Lysotracker Red (A) and Lysotracker Green (B). Asterisk (*) denotes the third pyrrole ring. (C-L) Cells were stained with Lysotracker Red according to manufacturer's recommendations and imaged with a confocal scanning microscope before and after exposing the field to epifluorescence with a red excitation filter. Images show $(\mathbf{C}, \mathbf{H})$ differential interference contrast, $(\mathbf{D}, \mathbf{I})$ excitation with a $488 \mathrm{~nm}$ laser prior to epifluorescence exposure, (E, J) excitation with a $594 \mathrm{~nm}$ laser, (F, K) $488 \mathrm{~nm}$ excitation after a one-second exposure to epifluorescence, and $(\mathbf{G}$, L) a merge of $\mathbf{E}, \mathbf{F}$ and $\mathbf{J}, \mathbf{K}$. (M) Decay of red fluorescence corresponds to an increase in green fluorescence. Cells stained with Lysotracker Red were imaged with 488 and $594 \mathrm{~nm}$ lasers after one-second exposures to epifluorescence and the mean fluorescent intensity (MFI) was plotted as a percentage of maximum intensity (\% max). (N) Emission spectra were determined for Lysotracker Green (LTG) and Lysotracker Red before (LTR Pre) and after (LTR Post) illuminating the field with epifluorescence for 5 seconds and $30 \%$ transmission. 
with which Lysotracker Red is used [1-5]. In light of our findings, we recommend against Lysotracker Red staining for epifluorescence or confocal microscopy in conjunction with green fluorescent molecules to avoid spurious results. Certainly, data showing apparent colocalization of a green fluorescent molecule with Lysotracker Red should be interpreted cautiously, and should be combined with additional lysosomal markers. There are, however, clear examples in the literature where the phenomenon of photoconversion has not occurred and Lysotracker Red has been used successfully [6]. Thus, to discriminate green and red fluorescence signals, care must be taken to insure that the Lysotracker Red dye is not modified by epifluorescent exposure prior to imaging.

\section{Acknowledgments}

The authors thank John Inman (NIH) and Kenneth Kirk (NIH) for helpful discussions. E.C.F. is a National Institutes of Health-University of Oxford Biomedical Research Scholar. This research was supported by the Intramural Research Program of the National Institutes of Allergy and Infectious Diseases, National Institutes of Health, USA.

\section{Reference}

1 Li Y, Wandinger-Ness A, Goldenring JR, Cover TL. Clustering and redistribution of late endocytic compartments in response to Helicobacter pylori vacuolating toxin. Mol Biol Cell 2004; 15:1946-1959.

2 Mesa R, Salomon C, Roggero M, Stahl PD, Mayorga LS. Rab22a affects the morphology and function of the endocytic pathway. J Cell Sci 2001; 114:4041-4049.

3 Liu CH, Thangada S, Lee MJ, et al. Ligand-induced trafficking of the sphingosine-1-phosphate receptor EDG-1. Mol Biol Cell 1999; 10:1179-1190.

4 Ramirez A, Heimbach A, Grundemann J, et al. Hereditary parkinsonism with dementia is caused by mutations in ATP13A2, encoding a lysosomal type 5 P-type ATPase. Nat Genet 2006; 38:1184-1191.

5 He YY, Huang JL, Chignell CF. Cleavage of epidermal growth factor receptor by caspase during apoptosis is independent of its internalization. Oncogene 2006; 25:1521-1531.

6 Blander JM, Medzhitov R. Regulation of phagosome maturation by signals from toll-like receptors. Science 2004; 304:10141018 .

(Supplementary Information is linked to the online version of the paper on the Cell Research website.) 